\title{
The dry and carbon-poor inner disk of TW Hydrae: evidence for a massive icy dust trap
}

\author{
Arthur D. Bosman ${ }^{1, \star}$ and Andrea Banzatti ${ }^{2}$ \\ ${ }^{1}$ Leiden Observatory, Leiden University, PO Box 9513, 2300 RA Leiden, The Netherlands \\ e-mail: arbos@umich.edu \\ 2 Department of Physics, Texas State University, 749 N Comanche Street, San Marcos, TX 78666, USA
}

Received 5 September 2019 / Accepted 22 November 2019

\begin{abstract}
Context. Gas giants accrete their envelopes from the gas and dust of proto-planetary disks, and therefore it is important to determine the composition of the inner few astronomical units, where most giant planets are expected to form.

Aims. We aim to constrain the elemental carbon and oxygen abundance in the inner disk $(R<2.3 \mathrm{AU})$ of TW Hya and compare with the outer disk $(R>2.3 \mathrm{AU})$ where carbon and oxygen appear underabundant by a factor of approximately 50 .

Methods. Archival Spitzer-IRS and VLT-CRIRES observations of TW Hya were compared with a detailed thermo-chemical model, DALI. The inner disk gas mass and elemental $\mathrm{C}$ and $\mathrm{O}$ abundances were varied to fit the mid-infrared $\mathrm{H}_{2}$ and $\mathrm{H}_{2} \mathrm{O}$ line fluxes as well as the near-infrared CO line flux.

Results. Best-fitting models have an inner disk that has a gas mass of $2 \times 10^{-4} M_{\odot}$ with $\mathrm{C} / \mathrm{H} \approx 3 \times 10^{-6}$ and $\mathrm{O} / \mathrm{H} \approx 6 \times 10^{-6}$. The elemental oxygen and carbon abundances of the inner disk are about 50 times lower than in the interstellar medium and are consistent with those found in the outer disk.

Conclusions. The uniformly low volatile abundances imply that the inner disk is not enriched by ices on drifting bodies that evaporate. This indicates that drifting grains are stopped in a dust trap outside the water ice line. Such a dust trap would also form a cavity as seen in high-resolution submillimeter continuum observations. If $\mathrm{CO}$ is the major carbon carrier in the ices, dust needs to be trapped efficiently outside the CO ice line of $\sim 20$ AU. This would imply that the shallow submillimeter rings in the TW Hya disk outside of $20 \mathrm{AU}$ correspond to very efficient dust traps. The most likely scenario is that more than $98 \%$ of the CO has been converted into less volatile species, for example $\mathrm{CO}_{2}$ and $\mathrm{CH}_{3} \mathrm{OH}$. A giant planet forming in the inner disk would be accreting gas with low carbon and oxygen abundances as well as very little icy dust, potentially leading to a planet atmosphere with strongly substellar $\mathrm{C} / \mathrm{H}$ and $\mathrm{O} / \mathrm{H}$ ratios.
\end{abstract}

Key words. protoplanetary disks - line: formation - astrochemistry - stars: individual: TW Hya

\section{Introduction}

The elemental abundances of carbon and oxygen in protoplanetary disks are a vital input to planet formation models and combined with characterization of exoplanets can tell us about the formation history of those planets. The simple picture of elemental abundances changing statically at the ice lines of the main chemical species (e.g. Öberg et al. 2011) is being increasingly enriched by a number of modeling studies and observations. Chemical evolution can be efficient in changing the composition of the disk gas, changing the major carriers of carbon and oxygen (e.g., Eistrup et al. 2016; Schwarz et al. 2018; Bosman et al. 2018a). Furthermore, disk dynamics and dust evolution can efficiently transport the volatile component of the disk, changing the elemental composition of the gas and ice (Kama et al. 2016; Booth et al. 2017; Bosman et al. 2018a; Krijt et al. 2016, 2018).

As the interplay of the physical and chemical processes is complex, observations are needed to benchmark disk models and provide much needed input for planet formation models.

* Present address: Department of Astronomy, University of Michigan, 311 West Hall, 1085 S. University Avenue, Ann Arbor, MI 48109, USA.
Observations of $\mathrm{CO}$ and $\mathrm{H}_{2} \mathrm{O}$ focusing on the outer regions of proto-planetary disks show that their abundances are up to two orders of magnitude lower than expected, implying that chemical and physical processes are indeed modifying the abundances of these species (Hogerheijde et al. 2011; Favre et al. 2013; Bergin et al. 2013; Miotello et al. 2017; Du et al. 2017). Nevertheless, planet formation models primarily need the composition around and within the $\mathrm{H}_{2} \mathrm{O}$ ice line, as this is where we expect giant planets to form and accrete their atmospheres (e.g. Kennedy \& Kenyon 2008; Cridland et al. 2017; Dawson \& Johnson 2018).

Therefore, we aim to constrain the elemental abundances in the inner disk of TW Hya. The outer disk of TW Hya has been well studied and the elemental composition of the gas has been constrained from a variety of observations, including HD to trace the total disk mass, allowing for the measurement of absolute abundances (Hogerheijde et al. 2011; Bergin et al. 2013; Kama et al. 2016; Schwarz et al. 2016; Trapman et al. 2017). These efforts have shown that both the volatile carbon and oxygen abundance in the outer disk are lowered by a factor of about 50 compared to the interstellar medium (ISM). Observations of ${ }^{13} \mathrm{C}^{18} \mathrm{O}$ in TW Hya show that while some $\mathrm{CO}$ comes from the 
Table 1. $\mathrm{H}_{2}$, CO, and $\mathrm{H}_{2} \mathrm{O}$ fluxes from observations and modeling.

\begin{tabular}{|c|c|c|c|c|c|}
\hline Molecular & Transition $^{(1)}$ & Wavelength $(\mu \mathrm{m})$ & Observed flux & Model flux ${ }^{(2)}$ & $E_{\text {up }}(\mathrm{K})$ \\
\hline $\mathrm{H}_{2}$ & $J=3-1(S(1))$ & 17.0 & $1.57 \pm 0.05$ & 1.7 & 1015 \\
\hline $\mathrm{H}_{2}$ & $J=4-2(S(2))$ & 12.3 & $1.21 \pm 0.20$ & 0.75 & 1681 \\
\hline $\mathrm{CO}$ & $v, J=1,10-0,11(v 1 P(10))$ & 4.75 & $0.64 \pm 0.05$ & 0.72 & 3330 \\
\hline $\mathrm{H}_{2} \mathrm{O}$ & $12_{5,8}-11_{2,9}$ & 17.12 & $<0.17$ & 0.03 & 3243 \\
\hline $\mathrm{H}_{2} \mathrm{O}$ & $11_{3,9}-10_{0,10}$ & 17.22 & $<0.29$ & 0.05 & 2473 \\
\hline $\mathrm{H}_{2} \mathrm{O}$ & $11_{2,9}-10_{1,10}$ & 17.36 & $<0.32$ & 0.11 & 2396 \\
\hline $\mathrm{H}_{2} \mathrm{O}$ & $9_{2,7}-8_{1,8}$ & 21.85 & $2.8 \pm 0.2$ & 6.7 & 1730 \\
\hline \multirow[t]{2}{*}{$\mathrm{H}_{2} \mathrm{O}$} & $94,5-8,6$ & 28.23 & $2.1 \pm 0.1$ & 1.3 & 1929 \\
\hline & $9_{5,4}-84,5$ & & & & 2122 \\
\hline \multirow[t]{2}{*}{$\mathrm{H}_{2} \mathrm{O}$} & $8_{6,3}-7_{5,2}$ & 28.43 & $1.6 \pm 0.2$ & 1.2 & 2031 \\
\hline & $8_{6,2}-7_{5,3}$ & & & & 2031 \\
\hline \multirow[t]{2}{*}{$\mathrm{H}_{2} \mathrm{O}$} & $77,0-6_{6,1}$ & 28.6 & $1.7 \pm 0.1$ & 1.2 & 2006 \\
\hline & $7_{7,1}-6_{6,0}$ & & & & 2006 \\
\hline \multirow[t]{2}{*}{$\mathrm{H}_{2} \mathrm{O}$} & $6_{3,1}-5_{0,5}$ & 30.87 & $2.7 \pm 0.2$ & 1.8 & 934 \\
\hline & $8_{5,4}-7_{4,3}$ & & & & 1806 \\
\hline \multirow[t]{2}{*}{$\mathrm{H}_{2} \mathrm{O}$} & $6_{6,1}-5_{5,0}$ & 33 & $3.96 \pm 0.46$ & 4.0 & 1504 \\
\hline & $7_{5,2}-6_{4,3}$ & & & & 1524 \\
\hline
\end{tabular}

Notes. Observed CO line flux is taken from Banzatti et al. (2017), and $\mathrm{H}_{2}$ and $\mathrm{H}_{2} \mathrm{O}$ fluxes are extracted from the Spitzer-IRS spectra using the method in Banzatti et al. (2012). The table denotes the upper level energy of the dominant transitions of each blend according to our model. The $\mathrm{H}_{2} \mathrm{~S}(2)$ line is contaminated by an $\mathrm{OH}$ line; the contribution of the $\mathrm{OH}$ line was estimated by taking the average flux of two adjacent $\mathrm{OH}$ lines. ${ }^{(1)}$ In the case of $\mathrm{H}_{2} \mathrm{O}$, the dominant transition(s) of the feature. (2) In units of $10^{-14} \mathrm{erg} \mathrm{s}^{-1} \mathrm{~cm}^{-2}$.

grains within the $\mathrm{CO}$ ice line, the $\mathrm{CO}$ abundance stays a factor of about 20 lower than the ISM abundance (Zhang et al. 2017), implying that a lot of the carbon is trapped on the grains or is converted into other species.

The physical and chemical structure of the inner disk of TW Hya has also been studied in detail. It is the disk that has been resolved at the highest physical resolution with ALMA (Andrews et al. 2016) and together with abundant photometry and infrared interferometry, there is a clear picture of the inner disk structure of TW Hya (Andrews et al. 2012; Menu et al. 2014; Kama et al. 2016). In the infrared, high signal-to-noise ratio Spitzer-IRS and VLT-CRIRES spectra have been taken. These observations include $3.2 \mathrm{~km} \mathrm{~s}^{-1}$ resolution observations of the $\mathrm{CO} v=1-0$ rovibrational band at $4.7 \mu \mathrm{m}$, and detections of the $\mathrm{CO}_{2} 15 \mu \mathrm{m} Q$-branch, pure rotational lines lines of $\mathrm{H}_{2}, \mathrm{H}_{2} \mathrm{O}$, and $\mathrm{OH}$, as well as a number of atomic hydrogen lines (Najita et al. 2010; Pontoppidan et al. 2008). Modeling efforts have further constrained the inner disk gas mass using $\mathrm{H}_{2}$ (Gorti et al. 2011) as well as the $\mathrm{H}_{2} \mathrm{O}$ content of the inner disk (Zhang et al. 2013). These studies hint at abundances for $\mathrm{CO}$ and $\mathrm{H}_{2} \mathrm{O}$ in the inner disk that are lower than expected from inner disk chemistry for gas of ISM composition, implying that elemental carbon and oxygen are depleted relative to the ISM.

Here we build on these studies, using the better-constrained outer disk structure and composition from Trapman et al. (2017), with an updated inner disk gap from ALMA observations (Andrews et al. 2016). Using this disk structure and the thermochemical code DALI we constrain the elemental carbon and oxygen abundance in the inner disk of TW Hya.

\section{Methods}

The lines and features, and their fluxes, that we consider for our modeling comparison are tabulated in Table 1. The CO flux for TW Hya is taken from Banzatti et al. (2017) based on VLT-CRIRES (Kaeufl et al. 2004) spectra presented in Pontoppidan et al. (2008, program ID 179.C-0151). The fluxes for $\mathrm{H}_{2}$ and $\mathrm{H}_{2} \mathrm{O}$ are extracted from the Spitzer-IRS spectrum obtained from program GO 30300 using the method described in Banzatti et al. (2012). The spectrum has been published in Najita et al. (2010) and Zhang et al. (2013).

We start with the Dust and LInes (DALI, Bruderer et al. 2012; Bruderer 2013) model of Trapman et al. (2017) for TW Hya (see also Kama et al. 2016, model parameters are given in Table A.1). This model fits the SED, many far-infrared and submillimeter lines, and the ALMA ${ }^{12} \mathrm{CO} J=3-2$ image. The most important lines are the HD 112 and $56 \mu \mathrm{m}$ lines, constraining the outer disk mass, and many CO (isotopologue) lines constraining the carbon abundance. On top of these, some atomic carbon and oxygen fine-structure lines have also been fit, which constrain the elemental carbon and oxygen abundances in the outer disk at $\mathrm{C} / \mathrm{H}=2.7 \times 10^{-6}$ and $\mathrm{O} / \mathrm{H}=5.8 \times 10^{-6}$ which are a factor 50 lower than expected for the ISM. In this work we update the inner disk structure moving the outer edge of the gap from 4 to $2.4 \mathrm{AU}$ in accordance with the bright submillimeter ring seen by Andrews et al. (2016) at this radius (assuming a distance of 54 parsec).

Figure 1 shows the surface density structure in the inner disk. The inner disk mass is varied by varying $\delta_{\text {gas }}$ in the inner disk using a model that has constant, low elemental abundances. The predicted $\mathrm{H}_{2}$ lines for DALI are compared to the observed fluxes. The model that fits best is then used to constrain the elemental $\mathrm{C}$ and $\mathrm{O}$ abundances. We make the simplifying assumptions that the $\mathrm{CO}$ abundance in the inner disk scales linearly with the total elemental carbon abundance, that the $\mathrm{H}_{2} \mathrm{O}$ abundance scales with the elemental oxygen that is not locked in $\mathrm{CO}$, and that changing the $\mathrm{CO}$ and $\mathrm{H}_{2} \mathrm{O}$ abundances does not significantly alter the gas temperatures. The elemental $\mathrm{C}$ and $\mathrm{O}$ abundances are varied by changing the $\mathrm{CO}$ and $\mathrm{H}_{2} \mathrm{O}$ abundances by a factor $\delta_{\mathrm{C}}$ and $\delta_{\mathrm{O}}$, respectively, above gas temperatures of $T_{\text {step }}$ of 70 , 150 , and $500 \mathrm{~K}$. Here, 70 and $150 \mathrm{~K}$ are the sublimation temperatures of $\mathrm{CO}_{2}$ and $\mathrm{H}_{2} \mathrm{O}$ respectively, whereas $500 \mathrm{~K}$ is a rough transition temperature for the release of carbon from a more refractory reservoir. The excitation is recalculated for these new 


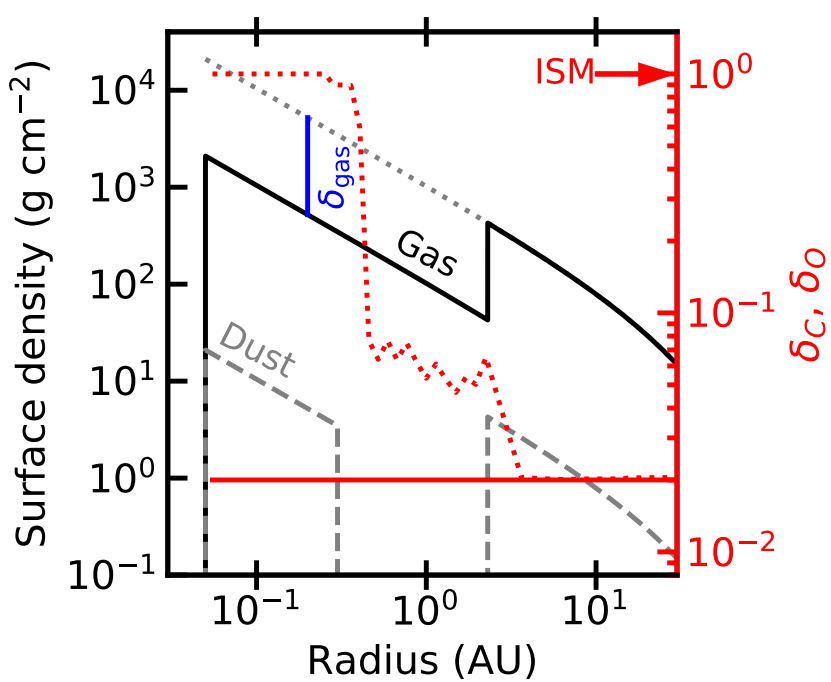

Fig. 1. Surface density structure in inner regions of the TW Hya model. $\delta_{\text {gas }}$ is the inner disk drop in gas surface density. The value $\delta_{\text {gas }}=0.1$ used here best fits the $\mathrm{H}_{2}$ observations. The red curves show the column averaged oxygen and carbon depletion factors in TW Hya. The red solid line shows a model with constant depletion. The dotted line shows the depletion profile assuming that carbon and oxygen return to the ISM values above $T_{\text {gas }}=150 \mathrm{~K}$.

abundance structures and the $\mathrm{CO} v 1 \mathrm{P}(10)$ line flux and the full $\mathrm{H}_{2} \mathrm{O}$ spectrum between 12 and $34 \mu \mathrm{m}$ is extracted.

The excitation for $\mathrm{H}_{2}$ is done in local thermodynamic equilibrium (LTE) as there are no collisional rate coefficients available for $\mathrm{H}_{2}$; however, the low Einstein A coefficients of the $\mathrm{H}_{2}$ lines mean that deviations from LTE are expected to be negligible (Wolniewicz et al. 1998). For $\mathrm{H}_{2} \mathrm{O}$ and $\mathrm{CO}$ the local excitation-de-excitation balance is calculated explicitly. For CO, collision rate coefficients from Yang et al. (2010) for $\mathrm{H}_{2}$ and Song et al. (2015) and Walker et al. (2015) for $\mathrm{H}$ are used (see also Bosman et al. 2019). For $\mathrm{H}_{2} \mathrm{O}$, the data file from the LAMDA database $^{1}$ (Schöier et al. 2005) is used.

\section{Results}

Figure 2 compares the emitting regions of the different species in the constant abundance model with $\delta_{\text {gas }}=0.1$. There is a large overlap in the emitting areas, especially in the inner disk $(<2.4 \mathrm{AU})$. Water has the most confined emitting area and only probes the inner disk while $\mathrm{CO}$ and $\mathrm{H}_{2}$ both also probe the outer disk, where the composition of the gas is already strongly constrained (Bergin et al. 2013; Kama et al. 2016; Trapman et al. 2017; Zhang et al. 2017).

Figure 3 compares the results of the DALI modeling with the observed fluxes. The $\mathrm{H}_{2}$ fluxes constrain the inner disk gas mass to be around $1.7 \times 10^{-4} M_{\odot}\left(\delta_{\text {gas }} \approx 0.1\right)$, comparable to the value found in Gorti et al. (2011). The observations show a higher $\mathrm{H}_{2} \mathrm{~S}(2) / \mathrm{S}(1)$ line ratio compared to the models, indicating that the average gas temperature in the inner disk of the model is too low. The upper level energies of the $\mathrm{CO}(\sim 3000 \mathrm{~K})$ and $\mathrm{H}_{2} \mathrm{O}$ $(\sim 1500 \mathrm{~K})$ are similar to or higher than the upper level energies of the $\mathrm{H}_{2}$ lines $(\sim 1000$ and $\sim 1700 \mathrm{~K})$, therefore a higher temperature in the inner disk would lead to even lower inferred values for $\mathrm{C} / \mathrm{H}$ and $\mathrm{O} / \mathrm{H}$.

\footnotetext{
1 http://strw.leidenuniv.nl/ moldata
}

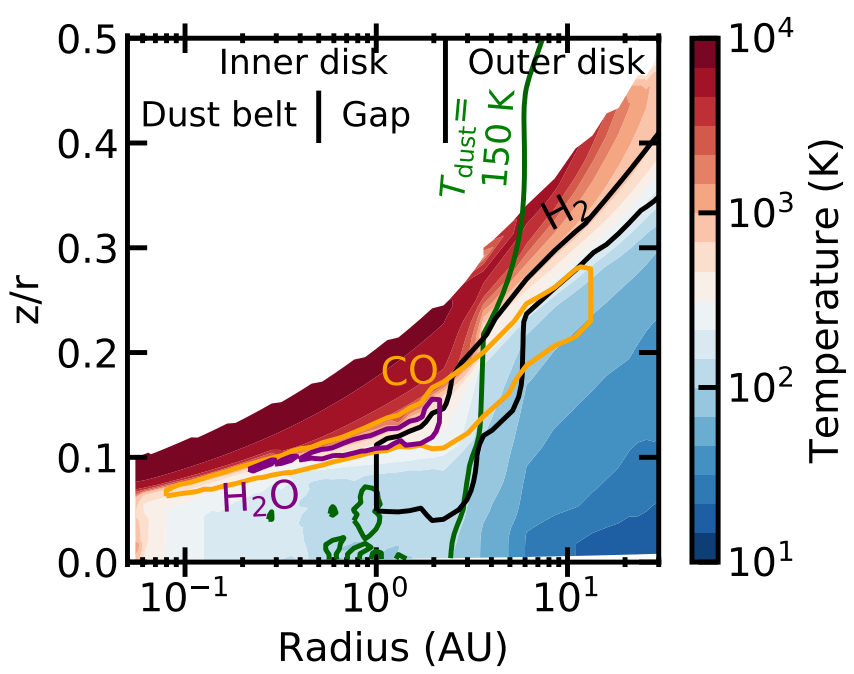

Fig. 2. Map of the gas temperature in the inner region of the model together with the emitting areas of the $\mathrm{H}_{2} \mathrm{~S}(1)$ line (black) the $\mathrm{CO} v 1$ vibrational lines (orange) and the strongest line of the $\mathrm{H}_{2} \mathrm{O} 33 \mu \mathrm{m}$ feature (purple). The green line shows $T_{\text {dust }}=150 \mathrm{~K}$, the approximate location of the $\mathrm{H}_{2} \mathrm{O}$ ice line. The emitting areas radially overlap in the inner disk.

Both the $\mathrm{CO}$ lines and $\mathrm{H}_{2} \mathrm{O}$ lines fit well to an inner disk elemental abundance similar to the abundance found in the outer disk, which is a factor 50 lower compared to the ISM. Even a jump of a factor of two in the elemental carbon or oxygen abundance above $500 \mathrm{~K}$ can be ruled out based on the observed fluxes. The $\mathrm{H}_{2} \mathrm{O}$ fluxes in Table 1 show that this model underpredicts the $\mathrm{H}_{2} \mathrm{O}$ detections between 20 and $31 \mu \mathrm{m}$ by a factor of about two, which is in line with the gas temperature in the model being too low.

\section{Discussion}

\subsection{Constraining the inner disk chemical structure}

Here, using a more complete physical and chemical structure of both the inner and the outer disk, we can quantify the total $\mathrm{H}_{2}$ mass, as well as the volatile carbon and oxygen abundance in the inner disk, confirming that the inner disk of TW Hya is both oxygen- and carbon-poor by a factor about 50 compared to the ISM. Furthermore, our modeling shows that there is no significant (factor 2 or more) increase in volatile carbon or oxygen in the inner disk. Thus, there is no sign of volatile release at the $\mathrm{CO}_{2}$ or $\mathrm{H}_{2} \mathrm{O}$ ice lines nor is there evidence of carbonaceous or silicate grain destruction at $T<500 \mathrm{~K}$.

Both Gorti et al. (2011) and Zhang et al. (2013) studied the inner region of TW Hya using detailed modeling. They note that they overproduce the $\mathrm{CO}$ rovibrational lines by a factor of about two. Furthermore, Gorti et al. (2011) assume LTE excitation for $\mathrm{CO}$, which generally underpredicts fluxes compared to models that include infrared pumping of the vibrational levels by the inner disk continuum emission (Bruderer et al. 2015; Bosman et al. 2017). These two factors together explain why our models need a $\mathrm{CO}$ or elemental carbon abundance that is almost two orders of magnitude lower than that of the ISM to fit the CO rovibrational line.

Zhang et al. (2013) use a low abundance in the inner disk $x_{\mathrm{H}_{2} \mathrm{O}}<10^{-6}$ with a ring of high abundance $\mathrm{H}_{2} \mathrm{O}$ at $4 \mathrm{AU}$ (their ice line) to produce the Spitzer lines. Including such a ring and fitting the $33 \mu \mathrm{m}\left(E_{\text {up }}=1504 \mathrm{~K}\right)$ feature would lead to inferring 

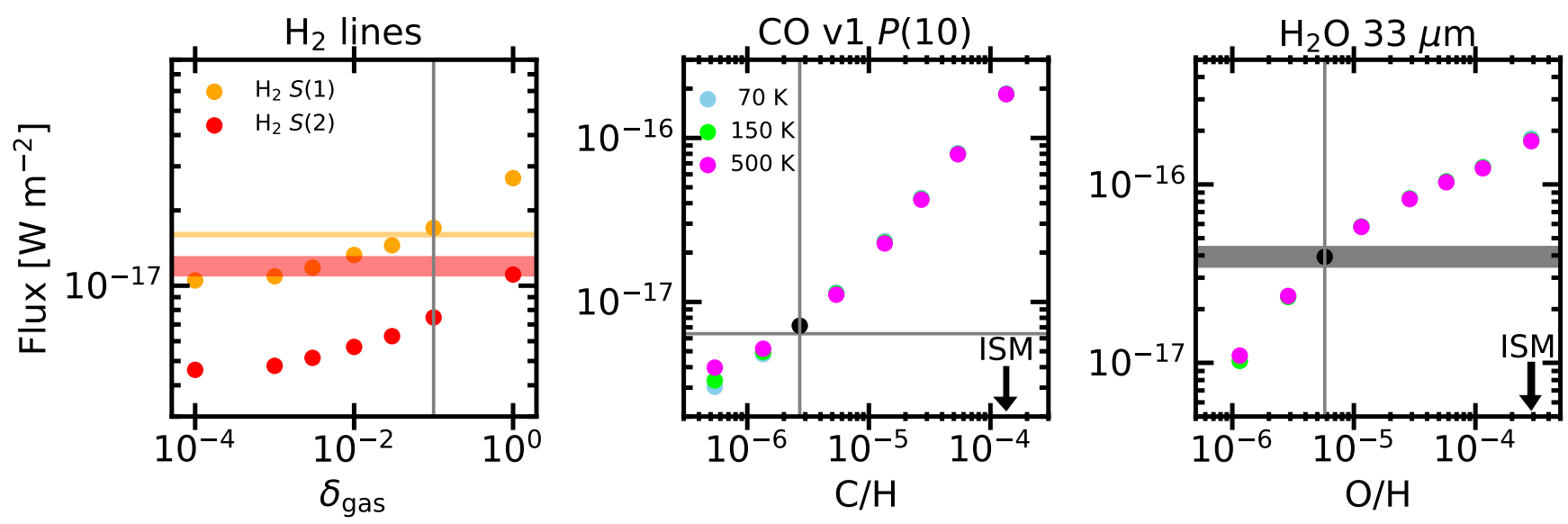

Fig. 3. Comparison of the $\mathrm{H}_{2}$ line fluxes (left), CO rovibrational line flux (middle), and the $\mathrm{H}_{2} \mathrm{O} 33 \mu \mathrm{m}$ feature flux (right) between models and data. Left panel: the amount of gas within $2.4 \mathrm{AU}$ is varied. Middle and right panels: $\delta_{\text {gas }}=0.1$ is used and $\mathrm{C} / \mathrm{H}$ and $\mathrm{O} / \mathrm{H}$ are varied respectively. Horizontal bands show the $1 \sigma$ variation of the observed fluxes. The different coloured points denote different values for $T_{\text {step }}$. The temperature threshold above which the abundances are varied. The vertical lines in the middle and right panel show the volatile carbon and oxygen abundances assumed for the ISM (Meyer et al. 1998; Lacy et al. 2017).

lower oxygen abundances in the inner disk; however, it would also move the $\mathrm{H}_{2} \mathrm{O}$ emission to colder gas, increasing the discrepancy between model and observations for the 22 and $28 \mu \mathrm{m}$ features. A constant-abundance model therefore fits the data better than a ring model.

\subsection{Hiding $C$ and $O$ carriers?}

The DALI models predict that $\mathrm{CO}$ and $\mathrm{H}_{2} \mathrm{O}$ are the dominant gas-phase oxygen and carbon carriers in the inner disk. However, it is possible that carbon and oxygen are locked in other gaseous species. There are a few obvious candidates for hiding more carbon and oxygen in molecular gas: $\mathrm{CO}_{2}, \mathrm{C}_{2} \mathrm{H}_{2}, \mathrm{HCN}$, and $\mathrm{CH}_{4}$. There is a $\mathrm{CO}_{2}$ feature detected in the Spitzer spectrum of TW Hya. Applying the model results from Bosman et al. (2017) to the detected feature retrieves a $\mathrm{CO}_{2}$ abundance lower than $10^{-9}$ with respect to $\mathrm{H}_{2}$. The models in Bosman et al. (2017) do not have a gap, which if included would only increase the strength of the $\mathrm{CO}_{2}$ lines. As such the $\mathrm{CO}_{2}$ abundance $<10^{-9}$ is a stringent upper limit and this molecule is not an abundant carrier of either carbon or oxygen.

$\mathrm{C}_{2} \mathrm{H}_{2}$ and $\mathrm{HCN}$ are detected in many proto-planetary disks (Salyk et al. 2011), however neither are detected in the spectrum of TW Hya. As the $Q$-branches of $\mathrm{HCN}$ and $\mathrm{C}_{2} \mathrm{H}_{2}$ around $14 \mu \mathrm{m}$ have similar upper level energies and Einstein A coefficients to the $15 \mu \mathrm{m} Q$-branch of $\mathrm{CO}_{2}$, both $\mathrm{HCN}$ and $\mathrm{C}_{2} \mathrm{H}_{2}$ should have been detected if they are as abundant as $\mathrm{CO}_{2}$. The lack of observed features implies that both $\mathrm{HCN}$ and $\mathrm{C}_{2} \mathrm{H}_{2}$ contain less than $1 \%$ of the volatile carbon. The final possible gaseous carbon carrier is $\mathrm{CH}_{4}$. Observations of this molecule have so far only been successful in one disk, in which $\mathrm{CH}_{4}$ lines have been detected in absorption (Gibb \& Horne 2013). The $\mathrm{CH}_{4}$ abundance is therefore not strongly constrained by observations. However, from chemical models $\mathrm{CH}_{4}$ is not expected to be the most abundant carbon carrier in inner disk atmospheres (Walsh et al. 2015; Agúndez et al. 2018). As such, with CO and $\mathrm{H}_{2} \mathrm{O}$ we trace the bulk of gaseous carbon and oxygen in the inner disk.

\subsection{Implications of uniform depletion}

The low elemental abundances in the inner disk imply one of two things: either the entire disk is depleted in volatiles, implying dust grains with almost no ices, or volatiles are efficiently locked in ices on solids that are not transported through the disk.

To deplete the entire disk in volatiles, a period of very strong radial drift coupled with strong radial mixing is necessary (e.g., Booth et al. 2017). In such a scenario, the icy grains drift from the mass reservoir in the outer disk into the regions of the disk where the ices desorb. This leads to enhancements in $\mathrm{C} / \mathrm{H}$ and $\mathrm{O} / \mathrm{H}$ in the inner regions of the disk. After a few million years all of this high- $\mathrm{C} / \mathrm{H}$ and high $-\mathrm{O} / \mathrm{H}$ gas is accreted onto the star and the disk is left depleted in carbon and oxygen. However, a disk like this will also be strongly depleted in solid material as the grains that transport the ices inward will continue to drift into the star leading to dust depletions at least as high as the volatile element depletion. This scenario is therefore very unlikely for TW Hya, which has a massive dust component and a gas-to-dust ratio of around 100 (Bergin et al. 2013).

As efficient transport is excluded, this leaves $98 \%$ of the carbon and oxygen locked in solids that are not efficiently transported. Assuming that $\mathrm{CO}$ and $\mathrm{H}_{2} \mathrm{O}$ are the dominant oxygen and carbon carriers in the outer disk, it is necessary to stop $\mathrm{CO}$ from being transported over the $\mathrm{CO}$ ice line by a dust trap at a location larger than where the CO ice line is located. For TW Hya this would imply that one of the shallow millimeter continuum rings of TW Hya outside of $20 \mathrm{AU}$ corresponds to an efficient dust trap. An efficient dust trap at $>20$ AU should result in a disk with a large cavity. As dust is abundant down to $2.4 \mathrm{AU}$, a second dust trap would be necessary at that location, which would trap $\mathrm{H}_{2} \mathrm{O}$-rich grains.

In this scenario $\mathrm{H}_{2} \mathrm{O}$ would be far more efficiently locked in large grains than $\mathrm{CO}$ as the former is frozen out in a larger fraction of the disk. However, if gaseous $\mathrm{CO}$ is efficiently converted into less volatile species, especially $\mathrm{CO}_{2}$ and $\mathrm{CH}_{3} \mathrm{OH}$, then the $\mathrm{CO}$ - and $\mathrm{H}_{2} \mathrm{O}$-depletion fractions are likely to be more similar and an efficient dust trap around $20 \mathrm{AU}$ is no longer strictly necessary. The age of TW Hya of $10 \mathrm{Mr}$, is long enough to convert large amounts of CO into other species (Donaldson et al. 2016; Bosman et al. 2018b). In this case, the dust should not be allowed to pass the $\mathrm{CO}_{2}$ or $\mathrm{CH}_{3} \mathrm{OH}$ ice lines. These ice lines are at nearly the same location as the $\mathrm{H}_{2} \mathrm{O}$ ice line at the inner edge of the outer disk. As such, a dust trap at the innermost submillimeter ring would trap all of the icy $\mathrm{CO}_{2}, \mathrm{CH}_{3} \mathrm{OH}$, and $\mathrm{H}_{2} \mathrm{O}$ in the outer disk. A schematic representation of this is given in Fig. 4. 


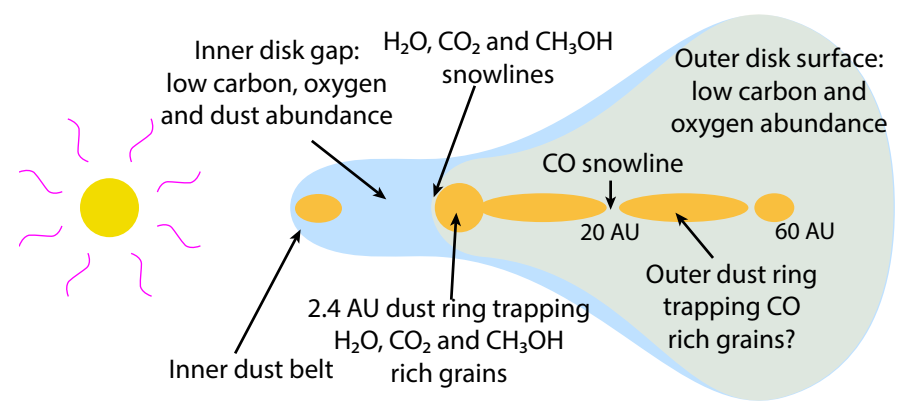

Fig. 4. Schematic of the TW Hya disk showing the locations that should trap the oxygen- and carbon-bearing molecules. Oxygen needs to be trapped outside of $2.4 \mathrm{AU}$ and is most likely trapped in the dust ring at that location. Carbon can either be trapped at the same location, with $\mathrm{CO}_{2}$ being the most probable carrier, or at larger radii outside the $\mathrm{CO}$ ice line, with $\mathrm{CO}$ being the most probable carrier.

In summary, we find that the elemental carbon and oxygen abundances in the inner disk are lower by a factor of approximately 50 compared to the ISM. Even at temperatures of $500 \mathrm{~K}$, the gaseous carbon or oxygen elemental abundances cannot have increased by a factor of two, strongly constraining the release of volatiles and grain destruction up to $500 \mathrm{~K}$. This is interpreted as the dust trap responsible for the dust-free cavity also trapping the major carbon- and oxygen-bearing ices outside of 2.4 AU. A planet currently accreting gas in the gap will accrete very low amounts of carbon and oxygen, while possibly accreting ISM concentrations of nitrogen and noble gasses. Depending on the accretion history this planet could have a substellar $\mathrm{C} / \mathrm{H}$ and $\mathrm{O} / \mathrm{H}$ abundance.

Acknowledgements. Astrochemistry in Leiden is supported by the Netherlands Research School for Astronomy (NOVA). This work is partly based on observations made with CRIRES on ESO telescopes at the Paranal Observatory under program ID 179.C-0151. This work is based in part on observations made with the Spitzer Space Telescope, which is operated by the Jet Propulsion Laboratory, California Institute of Technology under a contract with NASA. This project has made use of the SciPy stack (Virtanen et al. 2019), including NumPy (Oliphant 2006) and Matplotlib (Hunter 2007).

\section{References}

Agúndez, M., Roueff, E., Le Petit, F., \& Le Bourlot, J. 2018, A\&A, 616, A19 Andrews, S. M., Wilner, D. J., Hughes, A. M., et al. 2012, ApJ, 744, 162 Andrews, S. M., Wilner, D. J., Zhu, Z., et al. 2016, ApJ, 820, L40 Banzatti, A., Meyer, M. R., Bruderer, S., et al. 2012, ApJ, 745, 90 Banzatti, A., Pontoppidan, K. M., Salyk, C., et al. 2017, ApJ, 834, 152 Bergin, E. A., Cleeves, L. I., Gorti, U., et al. 2013, Nature, 493, 644

Booth, R. A., Clarke, C. J., Madhusudhan, N., \& Ilee, J. D. 2017, MNRAS, 469, 3994

Bosman, A. D., Bruderer, S., \& van Dishoeck, E. F. 2017, A\&A, 601, A36

Bosman, A. D., Tielens, A. G. G. M., \& van Dishoeck, E. F. 2018a, A\&A, 611, A80

Bosman, A. D., Walsh, C., \& van Dishoeck, E. F. 2018b, A\&A, 618, A182

Bosman, A. D., Banzatti, A., Bruderer, S., et al. 2019, A\&A, 631, A133

Bruderer, S. 2013, A\&A, 559, A46

Bruderer, S., van Dishoeck, E. F., Doty, S. D., \& Herczeg, G. J. 2012, A\&A, 541, A91

Bruderer, S., Harsono, D., \& van Dishoeck, E. F. 2015, A\&A, 575, A94

Cridland, A. J., Pudritz, R. E., Birnstiel, T., Cleeves, L. I., \& Bergin, E. A. 2017, MNRAS, 469, 3910

Dawson, R. I., \& Johnson, J. A. 2018, ARA\&A, 56, 175

Donaldson, J. K., Weinberger, A. J., Gagné, J., et al. 2016, ApJ, 833, 95

Du, F., Bergin, E. A., Hogerheijde, M., et al. 2017, ApJ, 842, 98
Eistrup, C., Walsh, C., \& van Dishoeck, E. F. 2016, A\&A, 595, A83

Favre, C., Cleeves, L. I., Bergin, E. A., Qi, C., \& Blake, G. A. 2013, ApJ, 776, L38

Gibb, E. L., \& Horne, D. 2013, ApJ, 776, L28

Gorti, U., Hollenbach, D., Najita, J., \& Pascucci, I. 2011, ApJ, 735, 90

Hogerheijde, M. R., Bergin, E. A., Brinch, C., et al. 2011, Science, 334, 338

Hunter, J. D. 2007, Comput. Sci. Eng., 9, 90

Kaeufl, H. U., Ballester, P., Biereichel, P., et al. 2004, in Ground-based Instrumentation for Astronomy, eds. A. F. M. Moorwood, \& M. Iye, Proc. SPIE, 5492, 1218

Kama, M., Bruderer, S., van Dishoeck, E. F., et al. 2016, A\&A, 592, A83

Kennedy, G. M., \& Kenyon, S. J. 2008, ApJ, 673, 502

Krijt, S., Ciesla, F. J., \& Bergin, E. A. 2016, ApJ, 833, 285

Krijt, S., Schwarz, K. R., Bergin, E. A., \& Ciesla, F. J. 2018, ApJ, 864, 78

Lacy, J. H., Sneden, C., Kim, H., \& Jaffe, D. T. 2017, ApJ, 838, 66

Menu, J., van Boekel, R., Henning, T., et al. 2014, A\&A, 564, A93

Meyer, D. M., Jura, M., \& Cardelli, J. A. 1998, ApJ, 493, 222

Miotello, A., van Dishoeck, E. F., Williams, J. P., et al. 2017, A\&A, 599, A113

Najita, J. R., Carr, J. S., Strom, S. E., et al. 2010, ApJ, 712, 274

Öberg, K. I., Boogert, A. C. A., Pontoppidan, K. M., et al. 2011, ApJ, 740, 109

Oliphant, T. 2006, NumPy: A Guide to NumPy (USA: Trelgol Publishing)

Pontoppidan, K. M., Blake, G. A., van Dishoeck, E. F., et al. 2008, ApJ, 684, 1323

Salyk, C., Pontoppidan, K. M., Blake, G. A., Najita, J. R., \& Carr, J. S. 2011, ApJ, 731, 130

Schöier, F. L., van der Tak, F. F. S., van Dishoeck, E. F., \& Black, J. H. 2005, A\&A, 432, 369

Schwarz, K. R., Bergin, E. A., Cleeves, L. I., et al. 2016, ApJ, 823, 91

Schwarz, K. R., Bergin, E. A., Cleeves, L. I., et al. 2018, ApJ, 856, 85

Song, L., Balakrishnan, N., Walker, K. M., et al. 2015, ApJ, 813, 96

Trapman, L., Miotello, A., Kama, M., van Dishoeck, E. F., \& Bruderer, S. 2017, A\&A, 605, A69

Virtanen, P., Gommers, R., Oliphant, T. E., et al. 2019, ArXiv e-prints [arXiv:1907.10121]

Walker, K. M., Song, L., Yang, B. H., et al. 2015, ApJ, 811, 27

Walsh, C., Nomura, H., \& van Dishoeck, E. 2015, A\&A, 582, A88

Wolniewicz, L., Simbotin, I., \& Dalgarno, A. 1998, ApJS, 115, 293

Yang, B., Stancil, P. C., Balakrishnan, N., \& Forrey, R. C. 2010, ApJ, 718, 1062

Zhang, K., Pontoppidan, K. M., Salyk, C., \& Blake, G. A. 2013, ApJ, 766, 82

Zhang, K., Bergin, E. A., Blake, G. A., Cleeves, L. I., \& Schwarz, K. R. 2017, Nat. Astron., 1, 0130

\section{Appendix A: DALI model}

Table A.1. Parameters of the TW Hya model.

\begin{tabular}{lc}
\hline \hline \multicolumn{2}{c}{ Stellar parameters } \\
\hline Stellar mass & $0.74 M_{\odot}$ \\
Stellar luminosity & $1 L_{\odot}$ \\
X-ray luminosity & $10^{30} \mathrm{erg} \mathrm{s}^{-1}$ \\
\hline \multicolumn{2}{c}{ Disk parameters } \\
Disk mass & $0.025 M_{\odot}$ \\
Critical radius $\left(R_{\mathrm{c}}\right)$ & $35 \mathrm{AU}$ \\
Surface density slope $(\gamma)$ & 1 \\
Scale height at $R_{\mathrm{c}}\left(h_{\mathrm{c}}\right)$ & $0.1 \mathrm{rad}$ \\
Flaring angle $(\psi)$ & 0.3 \\
Inner radius & $0.05 \mathrm{AU}$ \\
Gap inner radius & $0.3 \mathrm{AU}$ \\
Gap outer radius & $2.4 \mathrm{AU}$ \\
Inner disk gas-to-dust & 100 \\
Inner disk gas depletion & {$\left[10^{-5}-1\right]$} \\
Small dust size & $0.005-1 \mu \mathrm{m}$ \\
Small dust fraction & 0.01 \\
Large dust size & $0.005-1000 \mu \mathrm{m}$ \\
Large dust fraction & 0.99 \\
Large dust settling factor & 0.2 \\
\hline
\end{tabular}

